\title{
Effect of solvent-dependent viscoelastic properties of chitosan membranes on the permeation of 2-phenylethanol
}

\author{
Sofia G. Caridade, Ricardo M.P. da Silva, Rui L. Reis, João F. Mano* \\ 3B's Research Group - Biomaterials, Biodegradables and Biomimetics, Department of Polymer Engineering, University of Minho, \\ Headquarters of the European Institute of Excellence on Tissue Engineering and Regenerative Medicine AvePark, \\ Zona Industrial da Gandra S. Cláudio do Barco 4806-909 Caldas das Taipas Guimarães, Portugal \\ IBB - Institute for Biotechnology and Bioengineering, PT Government Associated Laboratory, Braga, Portugal
}

\section{A R T I C L E I N F O}

Article history:

Received 26 August 2008

Accepted 4 September 2008

Available online 18 September 2008

\section{Keywords:}

Chitosan

Diffusion

Glass transition

Membranes

Swelling

\begin{abstract}
A B S T R A C T
The viscoelastic behaviour of chitosan was followed by dynamic mechanical analysis (DMA) while the sample was immersed in gradient compositions of water/ethanol mixtures. The swelling equilibrium of chitosan membranes, both crosslinked with genipin or not, increased linearly with the water content. Increasing the water content, it was simultaneously observed a peak in the loss factor (around 25 vol.\%) and a reduction of the storage modulus, which was attributed to the $\alpha$-relaxation of chitosan. This was the first time that the glass transition dynamics in a polymer was monitored in immersion conditions where the composition of the plasticizer in the bath is changed in a controlled way. The water content at which $\tan \delta$ presented a maximum increased with both increasing frequency and increasing crosslinking density. The permeability decreased steadily with the ethanol content, reaching very low values around the glass transition. Therefore we hypothesize that conformational mobility of the polymeric chains may play an important role in the diffusion properties of molecules trough polymeric matrices.

(c) 2008 Elsevier Ltd. All rights reserved.
\end{abstract}

\section{Introduction}

Chitosan is a natural-derived polysaccharide obtained from the $\mathrm{N}$-deacetylation of chitin, which major industrial source is crustaceans' shells (Cauchie, 2002). The chitin $N$-deacetylation process is often not complete, giving origin to copolymers of 2-acetamido-2deoxy-D-glucopyranose (GluNAc) and 2-amino-2-deoxy-D-glucopyranose (GluN) (Varum, Anthonsen, Grasdalen, \& Smidsrod, 1991). The molar fraction of GluNAc is defined as the degree of $\mathrm{N}$-acetylation (DA). The term chitosan refers to the copolymers that are soluble in dilute acidic aqueous solutions (for DA $<0.60$, approx.), oppositely to the insoluble chitin. Both physicochemical (Lamarque, Lucas, Viton, \& Domard, 2005; Rinaudo, 2006; Sorlier, Denuziere, Viton, \& Domard, 2001; Sorlier, Rochas, Morfin, Viton, \& Domard, 2003; Sorlier, Viton, \& Domard, 2002) and biological properties (Huang, Khor, \& Lim, 2004) of these copolymers are strongly dependent on the DA.

Chitosan can be processed in a sort of devices varying in shape and size, such as membranes, nanoparticles, microparticles/microspheres, gels, tablets and capsules (Khor \& Lim, 2003; Pillai \& Panchagnula, 2001). This versatile polysaccharide has been proposed for a broad range of industrial applications, including wastewater

\footnotetext{
* Corresponding author. Tel.: +351 253510904; fax: +351 253510909.

E-mail addresses: sofia.caridade@dep.uminho.pt (S.G. Caridade), ricardosilva@dep.uminho.pt (R.M.P. da Silva), rgreis@dep.uminho.pt (R.L. Reis), jmano@dep.uminho.pt (J.F. Mano).

URL: http://www.3bs.uminho.pt. (J.F. Mano).
}

treatment, food, agriculture, cosmetics, biotechnological processes (Kumar, 2000) and separation technologies (Devi, Smitha, Sridhar, \& Aminabhavi, 2005; Uragami, Matsuda, Okuno, \& Miyata, 1994), as well as, for medical applications, such as biomaterials (Silva, Silva, Coutinho, Mano, \& Reis, 2004; Lopez-Perez, Marques, da Silva, Pashkuleva, \& Reis, 2007), drug delivery (Malafaya, Silva, \& Reis, 2007; Prabaharan \& Mano, 2005) and tissue engineering (Baran, Tuzlakoglu, Salgado, \& Reis, 2004; Silva, Ducheyne, \& Reis, 2007; Tuzlakoglu, Alves, Mano, \& Reis, 2004). It can be also used as a precursor to produce other materials through chemical modification (Jayakumar, Prabaharan, Reis, \& Mano, 2005; Prabaharan \& Mano, 2006a).

Chitosan belongs to a wide class of materials that has been receiving a great deal of interest for medical and pharmaceutical applications, hydrogels, which consist on macromolecular networks swollen in water or biological fluids. Hydrogels physical properties are obviously highly dependent on the swelling behaviour. Crosslinking is a commonly used route to restrict the polymer network swelling and, consequently, to tailor hydrogel properties. In this work, some chitosan membranes were crosslinked with genipin, because it has been reported to be non-cytotoxic and to form stable and biocompatible crosslinked products (Mi, Tan, Liang, \& Sung, 2002). Genipin is found in the traditional Chinese medicine and it is extracted from gardenia fruit (Jin, Song, \& Hourston, 2004). The reaction mechanism of chitosan with genipin has been investigated by Fwu-Long Mi and co-workers (Mi, Sung, \& Shyu, 2000). 
Dynamic mechanical analysis, DMA, is an adequate tool to characterize the mechanical/viscoelastic properties of polymeric materials, typically in wide temperature ranges and along several frequency decades. We have previously investigated the solid-state rheological properties of chitosan films by performing DMA experiments while the sample was immersed in a liquid bath (Silva et al., 2004; Mano, 2008). Such non-conventional methodologies could be useful to probe how materials behave in more realistic conditions, relatively to their applications, and also to extract relevant information about the dynamic behaviour of materials under different liquid environments. A strong reduction of the membranes stiffness could be observed upon complete immersion in aqueous solutions, which was accompanied by a sharp peak in the loss factor $(\tan \delta)($ Mano, 2008). However, in a free swelling situation, membranes rapidly uptake the solvent, making unfeasible the task of separately observe the glass to rubber-like state transition and the variation of mechanical properties that is exclusively due to the solvent uptake.

A number of swollen gel systems are known to undergo a 1st order phase transition when immersed in miscible solvent/non-solvent liquids (Alenichev, Hanykova, \& Ilavsky, 2007; Ilavsky, 1982; Ilavsky, Sedlakova, Bouchal, \& Plestil, 1995). A sudden decrease in the gel volume occurs at a critical non-solvent concentration. This 1 st order phase transition is given by a change of the chain conformation (Alenichev et al., 2007) and should not be confused with the glass transition phenomena. Ilavsky et al. (Ilavsky, 1982; Ilavsky et al., 1995) found that, in poly(acrylamide) networks, the swelling was linearly related with the shear modulus according to the predicted rubber elasticity, even in the region of the critical non-solvent concentration. Although, they detected departures from this linearity at higher non-solvent activity due to the vitrification of the polymer, the glass transition phenomena was not studied in great detail.

In this work, the mechanical and viscoelastic properties were measured with samples immersed in a liquid milieu containing different water (solvent) and ethanol (non-solvent) contents, providing intermediate swelling equilibrium conditions. The motivation is that by controlling swelling water molecules will interact differently with the chitosan structure and different levels of plasticization could be achieved. The hypothesis is that a transition from a glassy to a rubbery-like state could be attained just by controlling the uptake of liquid by chitosan. This could provide relevant information about the dynamics of the chitosan chains and maybe could be useful to better understand how such glass transition could be linked to important practical aspects of such systems, such as permeability of molecules.

\section{Experimental}

\subsection{Materials}

Genipin (GP) and 2-phenylethanol (PE) obtained from Wako and Fluka, respectively, were used as received. All other reagents and solvents used were of reagent grade and were used without further purification.

Chitosan (CTS) from crab shells was purchased from Sigma-Aldrich. Chitosan was purified before use by dissolving in acetic acid and precipitating with $\mathrm{NaOH}$ (final $\mathrm{pH} \sim 8$ ). Subsequently, the suspension was sieved; the precipitate was thoroughly washed with distilled water and rinsed twice with ethanol for about $4 \mathrm{~h}$. The product was frozen at $-80^{\circ} \mathrm{C}$ and lyophilised. Finally, the obtained product was milled and the chitosan powder was dried at $60^{\circ} \mathrm{C}$ overnight. The degree of $\mathrm{N}$-deacetylation (DD) was found to be $78.7 \%$ by the first derivative ultraviolet spectrophotometry, using both glucosamine (GluN) and $N$-acetylglucosamine (GluNAc) stan- dards for calibration (da Silva, Mano, \& Reis, 2008). The molecular weight $\left(M_{\mathrm{v}}\right)$ was determined by viscometry in $\mathrm{CH}_{3} \mathrm{COOH} 0.5 \mathrm{M} /$ $\mathrm{NaCH}_{3} \mathrm{COO} 0.2 \mathrm{M}$, which was found to be $770 \mathrm{kDa}$ according to the Mark-Houwing theory $\left(k=3.5 \times 10^{-4} ; a=0.76\right)$ (Terbojevich, Cosani, \& Muzzarelli, 1996).

\subsection{Preparation of Chitosan membranes}

Chitosan membranes were prepared by solvent casting. First, chitosan was dissolved at $1 \mathrm{wt} . \%$ in $1 \mathrm{wt} . \%$ aqueous acetic acid $(160 \mathrm{ml})$. Then, $10 \mathrm{ml}$ of a $1.8 \times 10^{-2} \mathrm{M}$ genipin solution was added dropwise to the chitosan solution under stirring. Non-crosslinked chitosan membranes were prepared directly from the original chitosan solutions, adding no genipin solution. The solutions were cast in Petri dishes and a blue colour typical of the crosslinking reaction with genipin appeared some hours later.

The simplest mechanism (without genipin oligomerisation) proposed by Mi et al. (2000) for the reaction between the genipin and chitosan is depicted in Fig. 1, in which a crosslinked chitosan network is established. The crosslinking degree $(x)$ was defined as the reagents feed molar ratio, considering the stoichiometry of the expected crosslinking reaction:

$x(\%)=\frac{2 n_{\mathrm{GP}}}{n_{-\mathrm{NH}_{2}}} \times 100$

where $n_{\mathrm{GP}}$ and $n_{-\mathrm{NH}_{2}}$ are the molar amounts of genipin and chitosan amine groups. From this definition, the crosslinking degree was calculated to be $5 \%$, as described before (da Silva, Caridade, San Roman, Mano, \& Reis, 2008a). However, it should be noticed that the real crosslinking efficiency depends upon the chemical conversion and on the occurrence of other parallel reactions, which can form either any or longer crosslinks, respectively.

After drying the solutions at room temperature, both non-crosslinked (CTSO) and crosslinked (CTS5) chitosan membranes were peeled off and neutralised in a $0.1 \mathrm{M} \mathrm{NaOH}$ solution for about $10 \mathrm{~min}$, washed thoroughly with distilled water and dried again.

\subsection{Swelling of chitosan membranes in water/ethanol mixtures}

The swelling of chitosan membranes in mixtures of a non-solvent (ethanol) and a solvent (water) was determined by immersing<smiles>COC(=O)C1=C[OH+]C(O)C2C(C)=CCC12</smiles>

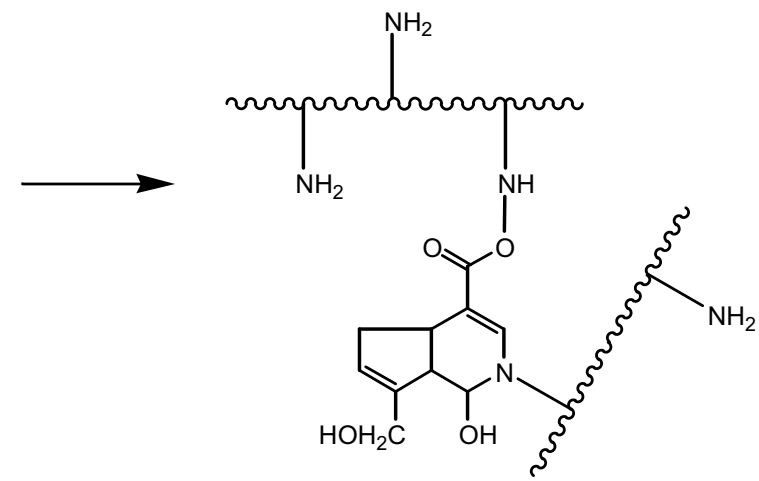

Fig. 1. Schematic representation of the crosslinking reaction of chitosan with genipin (Mi, Sung, \& Shyu, 2000). 
previously weighted chitosan membranes in mixtures of these solvents at compositions varying from pure water to pure ethanol. After around 4 and $48 \mathrm{~h}$, swelled samples were blotted with filter paper to remove the adsorbed solvent and weighted immediately. The swelling ratio $(S)$ was calculated using the following equation:

$S(w t . \%)=\frac{W-W_{0}}{W_{0}} \times 100$

where $W_{0}$ is the initial weight of the sample and $W$ is the weight of the swelled sample. Each swelling experiment condition was performed in triplicate.

\subsection{Dynamic mechanical analysis (DMA)}

All of the viscoelastic measurements were performed using a TRITEC2000B DMA from Triton Technology (UK), equipped with the tensile mode. The measurements were carried out at room temperature (ca. $20^{\circ} \mathrm{C}$ ). The distance between the clamps was $10 \mathrm{~mm}$ and the chitosan membrane samples were cut with about $2 \mathrm{~mm}$ width (measured accurately for each sample). Chitosan membranes were always analysed immersed in a liquid bath placed in a Teflon ${ }^{\circledR}$ reservoir. Chitosan membranes (CTS) were previously immersed in water/ethanol mixtures with different compositions until equilibrium was reached. The geometry of the samples was then measured and the samples were clamped in the DMA apparatus and immersed in the liquid with the same composition. After equilibration at room temperature (ca. $20^{\circ} \mathrm{C}$ ), the DMA spectra were obtained during a frequency scan between 0.1 and $40 \mathrm{~Hz}$. The experiments were performed under constant strain amplitude $(30 \mu \mathrm{m})$. A static pre-load of $1 \mathrm{~N}$ was applied during the tests to keep the sample tight.

Chitosan membranes (CTSO and CTS5) were also tested at constant frequency $(1 \mathrm{~Hz}$ and $10 \mathrm{~Hz}$ ) following the changes in the storage modulus $\left(E^{\prime}\right)$ and loss factor $(\tan \delta)$ as function of the water content. The dried samples of known geometry were first clamped in the DMA apparatus and immersed in a certain volume of ethanol $\left(V_{\mathrm{EtOH}}=400 \mathrm{ml}\right)$ and kept under the testing constant strain amplitude $(30 \mu \mathrm{m})$ during $30 \mathrm{~min}$. After this preconditioning step, $E^{\prime}$ reached an equilibrium value. Finally, the water was pumped into the reservoir at a constant flow rate $(Q=7.5 \mathrm{ml} / \mathrm{min})$, providing a time $(t)$ dependent change in the content of water described by the following equation:

Water $\left(\right.$ vol.\%) $=\frac{Q t}{Q t+V_{\mathrm{EtOH}}}$

Note that in these measurements $E^{\prime}$ should be taken as an apparent value as during introduction of water the geometry of the sample continuously changes due to swelling and the calculation of this parameter used the initial geometry of the sample.

\subsection{Permeability}

The permeability measurements were conducted using stoppered glass Franz-type diffusion cell (PermeGear ${ }^{\circledR}$ ). The volume of the receptor compartment was $8.1 \mathrm{ml}$ with an effective mass transfer area of $1 \mathrm{~cm}^{2}$. The membranes were previously equilibrated in the respective water/ethanol solvent mixture for $4 \mathrm{~h}$, placed between the two compartments and hold with a stainless steel clamp. The receptor compartment was immediately filled with the blank solvent mixture removing carefully the air bubbles. Finally, the donor compartment was filled with $2.0 \mathrm{ml}$ of 2-phenylethanol solution $(\sim 50 \mathrm{mM})$. Aliquots of $300 \mu \mathrm{l}$ were withdrawn from the receptor compartment at predetermined time periods and replenished by fresh solvent. The experiments were performed at room temperature $\left(\mathrm{ca} .20^{\circ} \mathrm{C}\right.$ ) and the receptor compartment was stirred at $400-600 \mathrm{rpm}$ using a magnetic bar to eliminate the boundary layer effect. The time dependent concentration of 2-phenylethanol in the receptor chamber was assessed by ultraviolet (UV) spectrophotometry using a Hellma ${ }^{\circledR}$ quartz microplate (96 wells) and a microplate reader from Synergy HT (Bio-tek ${ }^{\circledR}$ ). The absorbance was read at $258 \mathrm{~nm}$ and independent calibration curves were determined for each solvent composition over the entire range. The initial concentration of the donor solution was accurately assessed using the same method.

The diffusive mass transport is described by the Fick's first law:

$J=-D \frac{\mathrm{d} C}{\mathrm{~d} x}$

where $J$ is the flux, $D$ is the diffusion coefficient, $C$ is the solute concentration and $x$ is the distance in the direction perpendicular to the membrane. The simplest solution of this equation can be obtained at the steady-state, where flux is a constant and it can be maintained if the solute concentration is kept constant at the both donor $\left(C_{D}\right)$ and receptor $\left(C_{R}\right)$ half-cells. The Fick's first law may be then rewritten as:

$J=-D K \frac{\left(C_{D}-C_{R}\right)}{0-l}=\frac{P}{l}\left(C_{D}-C_{R}\right)$

where $K$ is the partition coefficient, $l$ is the membrane thickness and $P$ the permeability, defined as:

$P=D K$

The solute flux can be determined measuring the concentration in the receptor compartment, using the following equation:

$J=\frac{1}{A}\left(\frac{\mathrm{d} M_{R}}{\mathrm{~d} t}\right)$

where $M_{R}$ is total permeated mass accumulated in the receptor chamber at the time $t$ and $A$ is the membrane effective mass transfer area.

The initial permeation stage is termed the lag time, when the permeate is not detected in the receptor compartment. After the lag time, the concentration gradient inside the membrane is completely developed and a pseudo-steady-state permeation stage is observed. At this stage the concentration can be considered to vary linearly with time, this is, the flux is approximately constant (am Ende, Hariharan, \& Peppas, 1995; Kierstan et al., 2001; Matsuyama, Kitamura, \& Naramura, 1999). The assumption is valid for the sink conditions, which are maintained while $C_{D} \gg C_{R}$, that is:

$C_{D}-C_{R} \approx C_{D}$

The slope of the permeation curves was estimated by linear regression at that early pseudo-steady-state stage (correlation factor $>0.99$ ). The permeability, $P$, was then calculated combining and rearranging Eqs. (5)-(8) to obtain:

$P=\frac{1}{A}\left(\frac{\mathrm{d} M_{R}}{\mathrm{~d} t}\right) \frac{l}{C_{D}}$

where $C_{D}$ is the initial concentration at the donor compartment. The total permeated mass was calculated taking into consideration the concentration measured in the receptor chamber $\left(C_{R}\right)$, the volume of this chamber $\left(V_{R}\right)$ and also the amount of 2-phenylethanol withdrawn in each previous sampling aliquot.

\section{Results and discussion}

\subsection{Swelling of chitosan membranes in water/ethanol mixtures}

In the presence of suitable solvents, chemically or physically crosslinked polymer networks do not dissolve, but absorbs limited amounts of solvent swelling until the equilibrium is reached. On the other hand, non-solvents that are miscible with such polymer 
solvents might induce phase separation if added to a polymer solution at a critical concentration, a phenomenon known as cononsolvency. The combined effect of solvent and non-solvent in miscible liquid pairs is expected to be rather useful to control the swelling ratio within polymer networks. Chitosan, which is a semi-crystalline polymer, absorbs considerable amounts of water when immersed in aqueous environments (Silva et al., 2004). Moreover, several common organic solvents such as for instance acetone, ethanol or 2-propanol are typical chitosan non-solvents. In this work, ethanol was used as a non-solvent, aiming at provide control over the chitosan membranes swelling capability. The equilibrium swelling ratio $\left(S_{\text {eq }}\right)$ in water/ethanol mixtures was considered to have been achieved after $4 \mathrm{~h}$, since we did not found significant differences between the swelling ratio between 4 and $48 \mathrm{~h}$. Interestingly, the equilibrium swelling ratio varies linearly with the volumetric composition of the solvent mixture, as it can be observed in Fig. 2. A similar linear correlation was previously found for chitosan membranes immersed in 2-propanol/water mixtures (da Silva et al., 2008b; Uragami et al., 1994).

A number of polymer networks undergo a first order phasetransition when subjected to a small change in an external environmental parameter (e.g. temperature (Baysal \& Karasz, 2003), pH (Ceylan, Can, \& Okay, 2006) and solvent composition (Ilavsky et al., 1995)), at which an abrupt change in the volume is observed. Ilavsky and co-workers described polymeric networks in which a small variation in the composition of water/ethanol mixtures induces a jumpwise change in the volume of the gel, which is reflected in a simultaneous jumpwise change in the shear modulus (Ilavsky et al., 1995). However, they also found that in those systems the mechanical behaviour was predominantly determined by the degree of swelling, this is, the jumpwise change in the modulus adequately correlated with the jump in the swelling ratio. In the case under study, a continuous variation of the chitosan membranes swelling ratio is a strong indication that the referred type of volume transition do not occur under the experimental conditions. In this sense, if sharp variations in the mechanical or viscoelastic properties are detected, they should be more properly assigned to other type of molecular events (see next section).

It should be noticed that, although the chitosan membranes present a very low affinity to ethanol, as observed by an inexistent or non-detectable absorption at $100 \%$ ethanol, it does not mean that the composition of the solvent inside the membrane does not include ethanol at not null water concentrations. It fact, Yao et al. studied the swelling of chitosan-based hydrogels at different hydration degrees and they showed that water clusters coexist

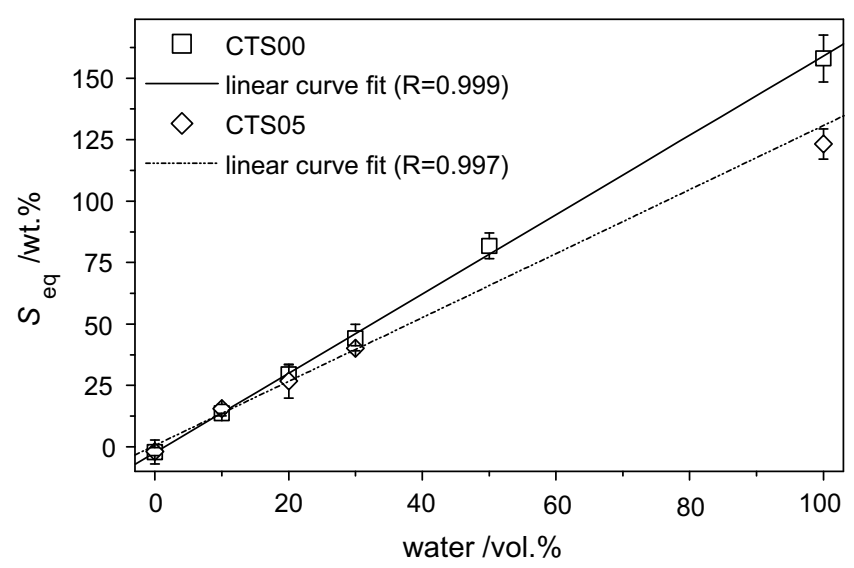

Fig. 2. Dependence on the water content of the equilibrium swelling ratio $\left(S_{\mathrm{eq}}\right)$ determined after immersion in water/ethanol mixture for non-crosslinked (CTSO) and crosslinked (CTS05) chitosan membranes. with water molecules hydrogen-bonded to the network at the beginning of swelling (Yao et al., 1998). This water clusters can constitute a route for ethanol entrance and permeation through the membrane. Nevertheless, chitosan membranes showed to be useful to break the azeotropic point of water/ethanol mixtures by evapomeation (Uragami et al., 1994) and of water/2-propanol mixtures by pervaporation (Devi et al., 2005), due to high water selectivity at lower water concentration. This indicates that the solvent composition inside the membrane is enriched in the water component and this effect is more pronounced for low amounts of water. The water selectivity of chitosan membranes used for pervaporation was reported to decrease steadily and to completely cease for water content higher than 40 wt.\% (Devi et al., 2005).

Finally, we assumed that similar viscoelastic phenomena should take place in both water and water/ethanol, since it is reasonable to consider that those phenomena are mainly related to the increase of the amount of non-bound absorbed solvent that can assist on the segmental mobility of the polymeric chains.

\subsection{Swelling-dependent viscoelastic properties}

Implanted devices in biomedical applications found a very hydrate environment, which may strongly influence their performance as compared with its dry state. In simulated physiological conditions chitosan can uptake up to $160 \%$ of water by weight, which strongly influence its mechanical properties (Silva et al., 2004). In a previous study the mechanical and viscoelastic properties of chitosan membranes were accessed in low hydration levels, just by changing the humidity of the environment of the material, or in complete immersed state (Mano, 2008). In the first case it was possible to follow by dynamic mechanical analysis, DMA, that the stiffness systematically decreased with increasing relative humidity but changes in the loss factor were much less evident. However, the strongest changes could be found upon complete immersion of the material in a liquid medium, where a reduction of stiffness by a factor higher than 50 could be observed, relatively to the dry state. At the moment of the immersion the decrease in the storage modulus was accompanied by a peak in the $\tan \delta$ when plotted the data as a function of time. It was suggested that this occurrence should be attributed to the occurrence of a glass transition that could be induced by the plasticizing effect of water that is being diffusing within the chitosan structure (Mano, 2008). In the dry state, hydrogen bonds are settled within the chitosan chains. When water comes inside the structure hydrogen bonds between water molecules and polar groups of chitosan will compete with the former ones and the conformational mobility of the chitosan chains may be enhanced. Therefore, upon a certain amount of water inside the amorphous fraction of chitosan a transition may take place from a glassy to a rubbery-like state. However, this hypothesis was never properly addressed. In a free swelling situation water uptake in chitosan takes place in less than $1 \mathrm{~min}$, and therefore it is not straightforward in an experimental point of view to investigate by DMA the corresponding changes in the viscoelastic properties. A possibility would be to control the swelling of the chitosan material by using mixtures of water and another miscible liquid that do not have molecular affinity with chitosan, such as some low molecular weight alcohols. It was seen before that different levels of swelling in chitosan could be achieved using different compositions of water and 2-propanol (da Silva et al., 2008b). This fact was used to impregnate different levels of a monomer in chitosan membranes and therefore to control the grafting level of the corresponding polymer that was synthesized in the polysaccharide structure. In this work chitosan films for DMA measurements were immersed in water/ethanol mixtures with different compositions until equilibrium was reached. The geometry of the samples was then measured and the samples were clamped in the DMA appara- 
tus and immersed in the liquid with the same composition. After equilibration at room temperature (ca. $20^{\circ} \mathrm{C}$ ), the DMA spectra were obtained during a frequency scan. The DMA results obtained with the different compositions are shown in Fig. 3. For a given mixture composition, the storage modulus (Fig. 3a) tends to increase with increasing frequency. For pure ethanol values of $E^{\prime}$ between 3 and 4 GPa could be found, that are very similar to the data found in dry chitosan (Mano, 2008). This provides a clear indication that ethanol exhibits a very little affinity with chitosan and in pure ethanol the chitosan membranes are in a very dehydrated form. This is in agreement with the swelling results, in which no absorption was detected for $100 \%$ ethanol. It can be then considered that the amorphous fraction of chitosan in this condition is in a glassy state. Oppositely, in 40 vol.\% water content values of $E^{\prime}$ about 80 times lower could be detected, also consistent with previous results obtained in wet chitosan (Mano, 2008). The inset of Fig. 3a shows the isochronal data at $1 \mathrm{~Hz}$, as a function of the water content in the mixture. It is clear that the storage modulus tend to decrease strongly as the mixture is enriched with water. This is consistent with the steady increase in swelling that was depicted in Fig. 2, but the shape of the variation of $E^{\prime}$ and water uptake is quite different: a linear relationship is observed in the later whereas a stronger decrease of $E^{\prime}$ could be detected around
25 vol.\% of water. These results clear demonstrate the importance of the swelling ratio of chitosan on the stiffness, which is relevant information for applications where the material may be found in different high hydration levels. Another important aspect is to look at the damping component of the viscoelastic performance of the chitosan membranes that could also have implications in their performance. Fig. $3 \mathrm{~b}$ shows the frequency dependence on the loss fac$\operatorname{tor}, \tan \delta$, for the same mixture compositions. Values between 0.03 and 0.3 could be found, indicating that water content of the swelling medium could have a profound impact on the capability of the material to absorb mechanical energy. For low levels of water content the $\tan \delta$ decreases with increasing frequency but above 25 vol.\% water content an opposite trend is observed. This is consistent with the existence of a relaxational process that takes place in the material upon the change in the mixture composition. The inset of Fig. 3b shows the isochronal cut of the results at $1 \mathrm{~Hz}$. One can detect a peak with a maximum of 25.4 vol.\% of water, that could be assigned with the glass transition phenomenon that was found before (Mano, 2008); such result is supported by the inflexion in the variation of $E^{\prime}$ with water vol.\% in the inset of Fig. 3a. It should be mentioned that in the previous study (Mano, 2008) the detection of the maximum in $\tan \delta$ was made in a system where one could not have the control of the swelling of the sample, and a

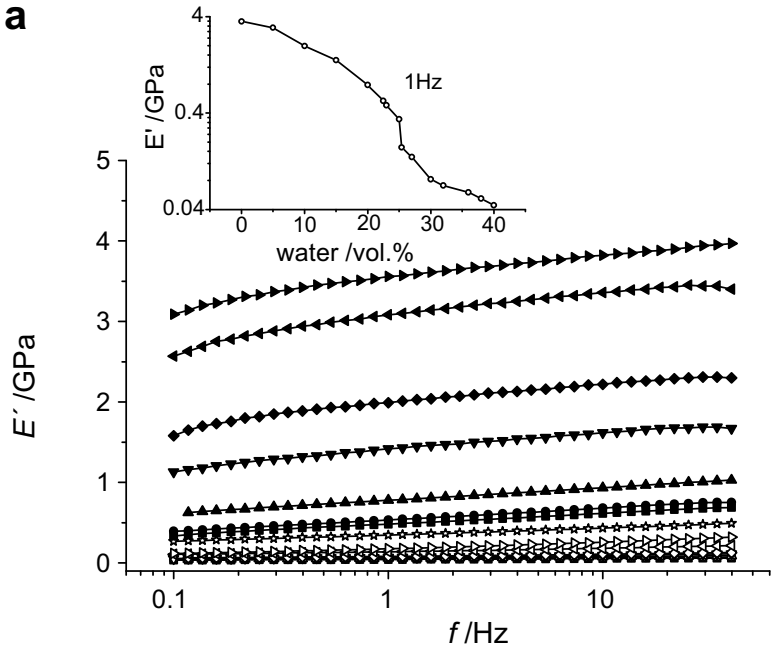

b

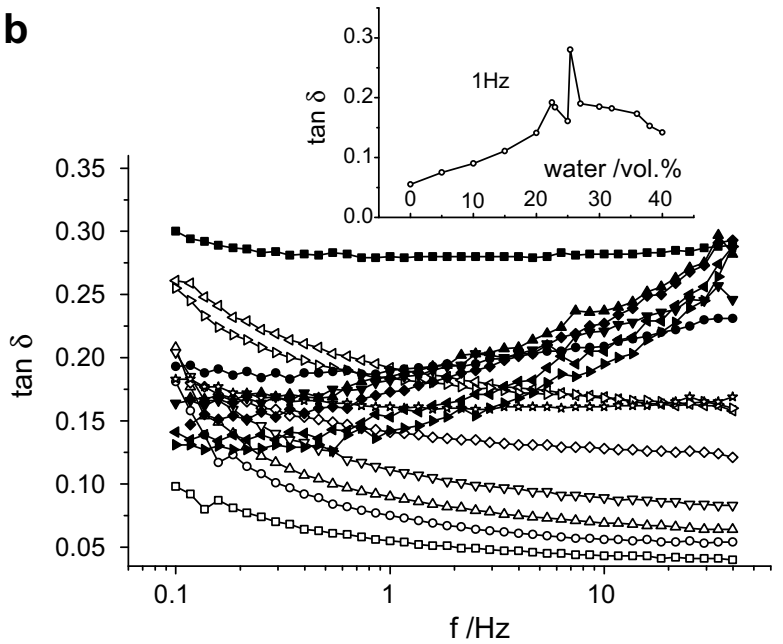

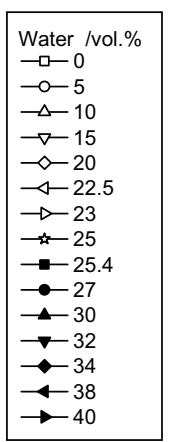

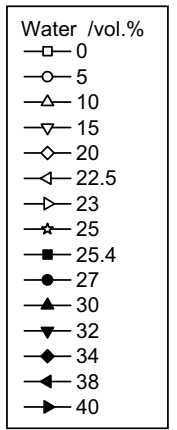

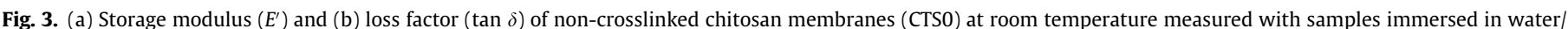

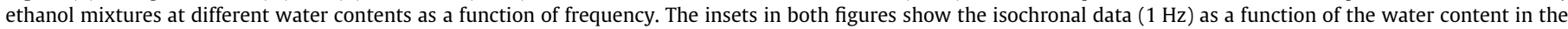
mixtures. 
in this case the glass transition process was detected in well controlled swelling measured in equilibrium.

Attempts were made in order to produce a master curve by shifting the DMA data obtained under different water contents along the $\log f$ scale, based on a possible time-swelling superposition principle. However, this was not possible because more than one effect influences the viscoelastic properties in this system: the introduction of more solvent in the structure will have a plasticizer effect, enhancing the conformational mobility of the polymeric segments; moreover, the introduction of more liquid in the structure will intrinsically decrease the stiffness of the structure and alter the viscoelastic properties of the material as one introduces a viscous element in it.

The glass transition process in chitosan has been addressed in different works, but the results are far from being in agreement. This is a not straightforward task as chitosan is a semi-crystalline biopolymers composed by very stiff chains, that could even form liquid crystalline structures in certain conditions (Prabaharan \& Mano, 2006b). Glass transition of chitosan, as in most of glass forming materials, has been only studied in the temperature axis, or combinations of temperature and frequency. In this context, some thermal analysis technique have been employed to characterize the $T_{\mathrm{g}}$ of chitosan, namely DMA and DSC: Mucha and Pawlak suggest that the glass transition of pre-heated chitosan can be found in the temperature range of $140-160{ }^{\circ} \mathrm{C}$ (Mucha \& Pawlak, 2005); however, similar DSC experiments did not present any clear change in the heat capacity in this temperature range (Kittur, Harish Prashanth, Udaya Sankar, \& Tharanathan, 2002). DSC measurements were also used by Cervera et al. to determine the $T_{\mathrm{g}}$ of chitosan that was situated between 130 and $139{ }^{\circ} \mathrm{C}$ (Cervera et al., 2004). Sakurai et al. used DSC and DMA and reported a $T_{\mathrm{g}}$ for chitosan at $203^{\circ} \mathrm{C}$, but again the identification is not completely clear (Sakurai, Maegawa, \& Takahashi, 2000). A close value for $T_{\mathrm{g}}$, around $195^{\circ} \mathrm{C}$, was reported by Shantha and Harding (2002) Pizzoli et al. also reported an high $T_{\mathrm{g}}$ values for chitosan, around $220^{\circ} \mathrm{C}$ (Pizzoli, Ceccorulli, \& Scandola, 1991). Attempts were previously made to perform DMA experiments on chitosan, also blended with starch or pullulan, previously exposed to different humidity levels (Lazaridou \& Biliaderis, 2002); however, during the temperature scans the equipments used did not allow the samples to be maintained at the desired hydration level, as in the case of the tests performed in the present work. In the work of Lazaridou and Biliaderis (2002) the $T_{\mathrm{g}}$ in dry chitosan was found to be around $95^{\circ} \mathrm{C}$, thus quite different from the other reported values. Other low values of $T_{\mathrm{g}}$ for chitosan were reported by Cheung et al., around $103^{\circ} \mathrm{C}$ as measured by DSC (Cheung, Wan, \& Yu, 2002), and by Toffey and Glasses, that suggested that it could range from 60 to $90^{\circ} \mathrm{C}$, as measured by DMA (Toffey \& Glasser, 2001). In a very complete study of chitosan/glycerol blends, Quijada-Garrido et al. detected by DMA a glass transition around $85^{\circ} \mathrm{C}$ (Quijada-Garrido, Iglesias-Gonzalez, Mazon-Arechederra, \& Barrales-Rienda, 2007). Dong et al. studied the glass transition of chitosan using different thermal analysis techniques (Dong, Ruan, Wang, Zhao, \& Bi, 2004); they reported values between 140 and $150{ }^{\circ} \mathrm{C}$, but the occurrence of the glass transition was not evident in most of the data, including dilatometry measurements, thermally stimulated current spectroscopy and even by DMA. Finally, the relaxational processes in chitosan were also analysed by dielectric relaxation spectroscopy, in neutralised and non-neutralised chitosan, also subjected to different thermal treatment to control water content (Viciosa, Dionisio, \& Mano, 2006; Viciosa, Dionisio, Silva, Reis, \& Mano, 2004). No evidences of an $\alpha$-relaxation was found up to $150^{\circ} \mathrm{C}$, that would be related to the dynamic glass transition. In that temperature region a process related to dc conductivity was detected, together with a Maxwell-Wagner-Sillars process, assigned to the accumulation of charges at the boundary of heterogeneities in the sample, at higher temperatures; these processes have a clear electrical nature and should not be visible by mechanical-related techniques. Therefore, the glass transition process as studied by dielectric relaxation spectroscopy in dry chitosan should occur above $150{ }^{\circ} \mathrm{C}$, and should be hidden by conductivity or other electrical effects.

To our knowledge the glass transition of polymeric samples was never systematically studied by DMA in which the composition of a liquid where the material is immersed is screened. Therefore another experimental setup was tested in which the chitosan sample was clamped in the DMA apparatus and immersed in an ethanol solution. The DMA parameters were then continuously monitored, in this case at two frequencies, 1 and $10 \mathrm{~Hz}$. During such measurements water was introduced in the reservoir at a constant flow rate, changing gradually the composition of the mixture. Fig. 4a shows the variation of the storage modulus and the loss factor as a function of the water composition in the liquid mixture of the bath. The variation of $E^{\prime}$ is quite similar with the trend displayed in Fig. 3a (inset). The $\tan \delta$ also presents a well defined peak around 25 vol.\% of water, also reaching values around 0.3 . Moreover, we can see that at $10 \mathrm{~Hz}$ the peak is shifted towards higher water contents. This strengthens the attribution of this process to a relaxational process in which the water content can be seen as an external variable that may control the molecular mobility of the polymeric structure. In this case one could consider the increase in water content to have a similar effect as a temperature increase or a frequency decrease in conventional DMA experiments. Note that the $\tan \delta$ peak of any relaxation shifts to higher temperatures when DMA measurements are collected at higher frequencies, being consistent with the results of Fig. 4a.
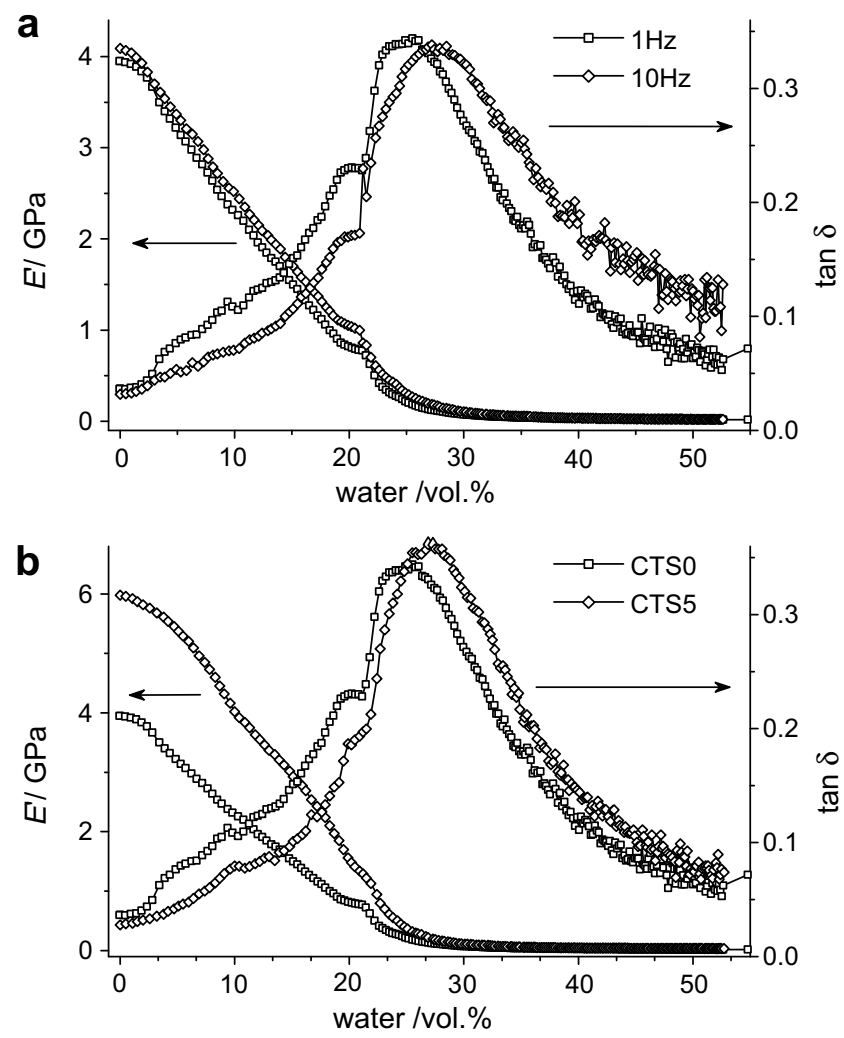

Fig. 4. Apparent storage modulus $\left(E^{\prime}\right)$ and loss factor $(\tan \delta)$ of at room temperature measured with samples immersed in water/ethanol mixtures as a function of water content: (a) dependence of the glass transition on frequency for non-crosslinked chitosan membranes (CTSO) and (b) on the crosslinking degree (CTS0 and CTS5) for $1 \mathrm{~Hz}$. 
In Fig. 4b similar results are presented for chitosan and crosslinked chitosan membranes. The crosslinked material exhibits higher values of $E^{\prime}$ as it would be expectable. A similar tendency was found before, in chitosan membranes crosslinked with glutaraldehyde (Silva et al., 2004). More interestingly is that the $\tan \delta$ peak is shifted to higher water contents for the crosslinked membrane. This is consistent with the decreasing of free volume caused by the chemical linkages, where more water is needed for the onset of cooperative motions. Such behaviour is also found in the $T_{\mathrm{g}}$ values of polymers prepared with different crosslinks. See, for example, the case of crosslinked poly (methylmethracrylate) networks (Alves, Ribelles, \& Mano, 2005; Alves, Ribelles, Tejedor, \& Mano, 2004).

It is also evident that in the left hand-side of the peaks of Fig. 4 a shoulder could be observed. This seems to be in agreement with the results found in the data of Fig. 3b that suggested that the process found in this region of water content exhibits a complex behaviour. One possibility could be related with the existence of a secondary relaxation for lower water contents, in which the material could be considered to be in the glassy state. Conventional DMA experiments have been performed before where beside the $\alpha$ relaxation a sub-glass process could be detected on dry samples. Guan, Liu, Zhang, and Yao (1998) reported the presence of a secondary $\beta$ process at $-55^{\circ} \mathrm{C}$ and a main $\alpha$-relaxation at $105^{\circ} \mathrm{C}$. Toffey and Glasses also detected similar processes (Guan et al., 1998). Mucha and Pawlak detected a $\beta$-relaxation in chitosan in the temperature range -20 to $-10{ }^{\circ} \mathrm{C}$, assigning that to local side chain movements of the polysaccharide (Mucha \& Pawlak, 2005). Finally, Quijada-Garrido et al. also reported two main processes in chitosan: a broad $\beta$-relaxation at $-30{ }^{\circ} \mathrm{C}$ (maximum of the $\tan \delta$ peak at $1 \mathrm{~Hz}$ ) and a complex $\alpha$-relaxation at $85^{\circ} \mathrm{C}$ (Toffey \& Glasser, 2001); the dynamic glass transition decreased with the increase content of glycerol, following a Gordon-Taylor-Woods model. Interestingly, the position of the secondary peak also decreased with the increase of glycerol content. In this case the $\beta$-relaxation was assigned to non-cooperative motions of the lateral groups linked to glycerol by hydrogen bonding.

In conclusion the changing in the composition of water in a water/ethanol mixture could be useful to detect the relaxational process that could be related to the glass transition. The main peak could correspond to an $\alpha$-relaxation, as seen in dynamic experiments as a function of temperature or frequency. However, it seems to be also possible to detect a secondary process that may be assigned to a $\beta$-relaxation. However, more experiments should be performed in other chitosan materials with different deacetylation degree or molecular weight in order to be more confident about this attribution. The occurrence of the glass transition seems to not affect the swelling capability of the material (see Fig. 2), although it could might influence the swelling kinetics toward the equilibrium. This process could have an influence on other processes where conformational mobility is relevant. In this context a series of permeability experiments on chitosan presenting different levels of swelling were carried out.

\subsection{Permeability}

The influence of swelling on the permeability of small molecular weight drugs was assessed using 2-phenylethanol as a non-ionic model solute. Typical curves of the fractional release showed a faster permeation rate through chitosan membranes for high water concentration (see Fig. 5a). In this case the molecular weight of the permeate is small enough to readily diffuse through the swollen network. Moreover, it is possible to observe an early permeation stage for a fractional release inferior than 0.2 that presents a linear profile, which corresponds to the pseudo-steady-state. This means that flux is constant within that time interval and that the sink conditions are maintained. The permeability was derived from the flux rate calculated at this region. It can be observed in Fig. $5 b$ that the permeability decreases sharply until it reaches very small values around the glass transition at 25 vol.\% of water.

Two effects may directly influence the permeability of the chitosan membranes in the different mixtures. The more obvious one is related with the total amount of liquid that was uptake by the film: as the water content in the mixture increase, more free water can be found within the membrane structure and the diffusion coefficient in the system increases, increasing the global permeability of 2-phenylethanol through the membrane. However conformational mobility of the polymeric chain also could play a role. Lin and Metters (2006) referred about the importance on the transition from the glassy to a rubbery-like state in the mobility of the polymeric chains of the matrix and the corresponding diffusivity of entrapped molecules. In the particular case of this work for sufficiently low water contents the average pore size of the network may be smaller than the volume of the molecules and segmental motions may be required in order that through fluctuations in the free volume, the molecules could find openings between the matrix chains large enough to allow their passage. In the glassy state, that in the present case should occur for water contents in the swelling medium below 25 vol.\%, such dynamic process ceases and the diffusion should be highly compromised, being consistent with the data in Fig. 5 .

\section{Conclusions}

The swelling capability of chitosan films can be controlled by using water/ethanol mixtures with different compositions. The viscoelastic properties of non-crosslinked and crosslinked chitosan samples immersed in different water/ethanol environments was assessed by dynamic mechanical analysis, DMA. The storage mod-
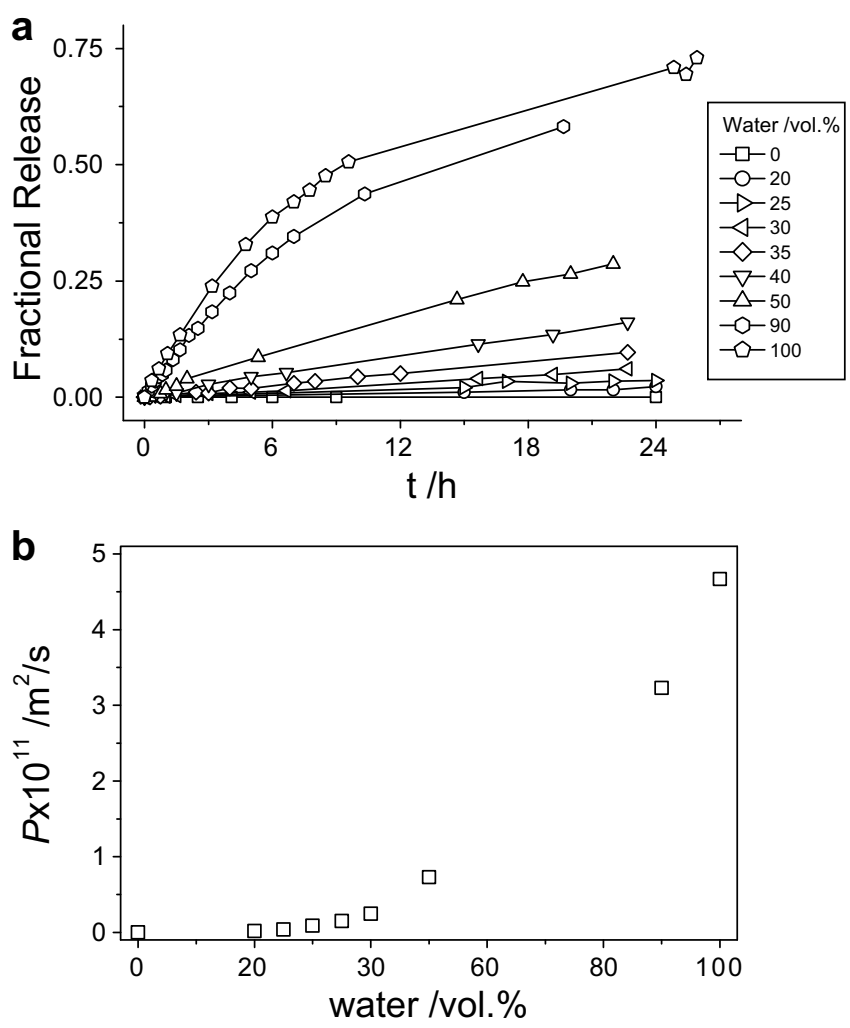

Fig. 5. Effect of the water content in (a) the fractional release profile and in (b) the permeability of non-crosslinked chitosan membranes (CTSO) 
ulus decreased with increasing water content; an inflection of this trend was coincident with the presence of a peak in the loss factor data, indicating that a relaxation process could be induced by enriching the solvent content in the chitosan structure, which was identified as a glass transition process. The position of the maximum of the $\tan \delta$ peak shift to higher water contents when frequency or crosslinking density increased. To our knowledge this constitutes the first attempt to study the glass transition by DMA of samples immersed in liquid mixtures composed by a solvent and a non-solvent. We believe that such plasticization effect induced by one of the component of the liquid mixture could be used to investigate the glass transition of other polymeric systems and hydrogels, including those having stiff chains such as polysaccharides. The glass to rubber-like state transition took place when the water content was about 25 vol.\%. For water/ethanol mixtures with less water contents the permeability of a low molecular weight model permeate was found to be very small; this could be explained by the fact that in the glassy state the conformational mobility is restricted, preventing free volume fluctuations and the corresponding diffusion of the molecules within the polymeric structure.

Such kind of experimental protocols may represent a new tool to study glass transition dynamics in polymeric systems, especially those were conformational mobility is difficult to detect without plasticization. In a more practical point of view such methodologies may provide useful information about diffusion and swelling relationships in the context of systems for sustained release of molecules (including drugs or other therapeutic substances) or membranes for separation processes. Water/alcohol (or other solvent/non-solvent) mixtures may be also interesting liquids for incorporating in a controlled way molecules inside chitosan-based systems, including drugs or monomers for posterior polymerization, in the case of inter-penetrating networks. As materials may be found in different hydration states in biomedical applications, DMA experiments in controlled swelling conditions may provide meaningful information of the mechanical performance of biomaterials.

\section{Acknowledgements}

This work was partially supported by the Portuguese Foundation for Science and Technology (FCT), through funds from the POCTI and/or FEDER programmes and through the scholarship SFRH/BPD/34545/2007 granted to Ricardo M.P. da Silva. This work was carried out under the scope of the European NoE EXPERTISSUES (NMP3-CT-2004-500283) and also partially supported by the EU funded projects HIPPOCRATES (STREP - NMP3-CT-2003505758) and PROTEUS (INTERREG III A - SP1.P151/03). The technical assistance of Ana Pinheiro in the execution of the DMA experiments is also acknowledged.

\section{References}

Alenichev, I., Hanykova, L., \& Ilavsky, M. (2007). Swelling and mechanical behavior of ionized poly(acrylamide-co- $N, N^{\prime}$-diethylacrylamide) gels in water/acetone mixtures and in water at various temperatures. Polymer Bulletin, 58(3), $575-586$.

Alves, N. M., Ribelles, J. L. G., \& Mano, J. F. (2005). Enthalpy relaxation studies in polymethyl methacrylate networks with different crosslinking degrees. Polymer, 46(2), 491-504.

Alves, N. M., Ribelles, J. L. G., Tejedor, J. A. G., \& Mano, J. F. (2004). Viscoelastic behavior of poly(methyl methacrylate) networks with different cross-linking degrees. Macromolecules, 37(10), 3735-3744.

am Ende, M. T., Hariharan, D., \& Peppas, N. A. (1995). Factors influencing drug and protein-transport and release from ionic hydrogels. Reactive Polymers, 25(2-3), 127-137.

Baran, E. T., Tuzlakoglu, K., Salgado, A. J., \& Reis, R. L. (2004). Multichannel mould processing of 3D structures from microporous coralline hydroxyapatite granules and chitosan support materials for guided tissue regeneration/ engineering. Journal of Materials Science - Materials in Medicine, 15(2), 161-165.
Baysal, B. M., \& Karasz, F. E. (2003). Coil-globule collapse in flexible macromolecules. Macromolecular Theory and Simulations, 12(9), 627-646.

Cauchie, H. M. (2002). Chitin production by arthropods in the hydrosphere. Hydrobiologia, 470(1-3), 63-96.

Cervera, M. F., Heinamaki, J., Rasanen, M., Maunu, S. L., Karjalainen, M., Acosta, O. M. N., et al. (2004). Solid-state characterization of chitosans derived from lobster chitin. Carbohydrate Polymers, 58(4), 401-408.

Ceylan, D., Can, V., \& Okay, O. (2006). Phase transition of acrylamide-based polyampholyte gels in water. Journal of Macromolecular Science Part a - Pure and Applied Chemistry, 43(10), 1635-1649.

Cheung, M. K., Wan, K. P. Y., \& Yu, P. H. (2002). Miscibility and morphology of chiral semicrystalline poly-(R)-(3-hydroxybutyrate)/chitosan and poly-(R)-(3hydroxybutyrate-co-3-hydroxyvalerate)/chitosan blends studied with DSC, H$1 \mathrm{~T}-1$ and T-1 rho CRAMPS. Journal of Applied Polymer Science, 86(5), 12531258.

da Silva, R. M. P., Mano, J. F., \& Reis, R. L. (2008). Straightforward determination of the degree of $\mathrm{N}$-acetylation of chitosan by means of the 1 st derivative UV spectrophotometry. Macromolecular Chemistry and Physics, 209(14), 14631472.

da Silva, R. M. P., Caridade, S. G., San Roman, J., Mano, J. F., \& Reis, R. L. (2008a). Transport of small anionic and neutral solutes through chitosan membranes: Dependence on crosslinking and chelation of divalent cations. Biomacromolecules, 9(8), 2132-2138.

da Silva, R. M. P., Lopez-Perez, P. M., Elvira, C., Mano, J. F., San Roman, J., \& Reis, R. L. (2008b). Poly( $N$-isopropylacrylamide) surface grafted chitosan membranes as a new substrate for cell sheet engineering and manipulation. Biotechnology and Bioengineering. doi:10.1002/bit.22004.

Devi, D. A., Smitha, B., Sridhar, S., \& Aminabhavi, T. M. (2005). Pervaporation separation of isopropanol/water mixtures through crosslinked chitosan membranes. Journal of Membrane Science, 262(1-2), 91-99.

Dong, Y. M., Ruan, Y. H., Wang, H. W., Zhao, Y. G., \& Bi, D. X. (2004). Studies on glass transition temperature of chitosan with four techniques. Journal of Applied Polymer Science, 93(4), 1553-1558.

Guan, Y. L., Liu, X. F., Zhang, Y. P., \& Yao, K. D. (1998). Study of phase behavior on chitosan/viscose rayon blend film. Journal of Applied Polymer Science, 67(12), 1965-1972.

Huang, M., Khor, E., \& Lim, L. Y. (2004). Uptake and cytotoxicity of chitosan molecules and nanoparticles: Effects of molecular weight and degree of deacetylation. Pharmaceutical Research, 21(2), 344-353.

Ilavsky, M. (1982). Phase-transition in swollen gels. 2. Effect of charge concentration on the collapse and mechanical-behavior of polyacrylamide networks. Macromolecules, 15(3), 782-788.

Ilavsky, M., Sedlakova, Z., Bouchal, K., \& Plestil, J. (1995). Phase-transition in swollen gels. 21. Effect of acrylamide quaternary-salts with various alkyl lengths on the collapse, mechanical, and saxs behavior of poly(acrylamide) networks. Macromolecules, 28(20), 6835-6842.

Jayakumar, R., Prabaharan, M., Reis, R. L., \& Mano, J. F. (2005). Graft copolymerized chitosan - present status and applications. Carbohydrate Polymers, 62(2), $142-158$

Jin, J., Song, M., \& Hourston, D. J. (2004). Novel chitosan-based films cross-linked by genipin with improved physical properties. Biomacromolecules, 5(1), 162-168.

Khor, E., \& Lim, L. Y. (2003). Implantable applications of chitin and chitosan. Biomaterials, 24(13), 2339-2349.

Kierstan, K. T. E., Beezer, A. E., Mitchell, J. C., Hadgraft, J., Raghavan, S. L., \& Davis, A. F. (2001). UV-spectrophotometry study of membrane transport processes with a novel diffusion cell. International Journal of Pharmaceutics, 229(1-2), 87-94.

Kittur, F. S., Harish Prashanth, K. V., Udaya Sankar, K., \& Tharanathan, R. N. (2002) Characterization of chitin, chitosan and their carboxymethyl derivatives by differential scanning calorimetry. Carbohydrate Polymers, 49(2), 185-193.

Kumar, M. N. V. R. (2000). A review of chitin and chitosan applications. Reactive $\mathcal{E}$ Functional Polymers, 46(1), 1-27.

Lamarque, G., Lucas, J. M., Viton, C., \& Domard, A. (2005). Physicochernical behavior of homogeneous series of acetylated chitosans in aqueous solution: Role of various structural parameters. Biomacromolecules, 6(1), 131-142.

Lazaridou, A., \& Biliaderis, C. G. (2002). Thermophysical properties of chitosan, chitosan-starch and chitosan-pullulan films near the glass transition. Carbohydrate Polymers, 48(2), 179-190.

Lin, C. C., \& Metters, A. T. (2006). Hydrogels in controlled release formulations: Network design and mathematical modeling. Advanced Drug Delivery Reviews, 58(12-13), 1379-1408.

Lopez-Perez, P. M., Marques, A. P., da Silva, R. M. P., Pashkuleva, I., \& Reis, R. L. (2007). Effect of chitosan membranes' surface modification via plasma induced polymerization on the adhesion of Osteoblast-like cells. Journal of Materials Chemistry, 17(38), 4064-4071.

Malafaya, P. B., Silva, G. A., \& Reis, R. L. (2007). Natural-origin polymers as carriers and scaffolds for biomolecules and cell delivery in tissue engineering applications. Advanced Drug Delivery Reviews, 59(4-5), 207-233.

Mano, J. F. (2008). Viscoelastic properties of chitosan with different hydration degrees as studied by dynamic mechanical analysis. Macromolecular Bioscience, 8, 67-76.

Matsuyama, H., Kitamura, Y., \& Naramura, Y. (1999). Diffusive permeability of ionic solutes in charged chitosan membrane. Journal of Applied Polymer Science, 72(3), 397-404.

Mi, F. L., Sung, H. W., \& Shyu, S. S. (2000). Synthesis and characterization of a novel chitosan-based network prepared using naturally occurring crosslinker. Journal of Polymer Science Part A-Polymer Chemistry, 38(15), 2804-2814. 
Mi, F. L., Tan, Y. C., Liang, H. F., \& Sung, H. W. (2002). In vivo biocompatibility and degradability of a novel injectable-chitosan-based implant. Biomaterials, 23(1), 181-191.

Mucha, M., \& Pawlak, A. (2005). Thermal analysis of chitosan and its blends. Thermochimica Acta, 427(1-2), 69-76.

Pillai, O., \& Panchagnula, R. (2001). Polymers in drug delivery. Current Opinion in Chemical Biology, 5(4), 447-451.

Pizzoli, M., Ceccorulli, G., \& Scandola, M. (1991). Molecular motions of chitosan in the solid-state. Carbohydrate Research, 222, 205-213.

Prabaharan, M., \& Mano, J. F. (2005). Chitosan-based particles as controlled drug delivery systems. Drug delivery, 12(1), 41-57.

Prabaharan, M., \& Mano, J. F. (2006a). Stimuli-responsive hydrogels based on polysaccharides incorporated with thermo-responsive polymers as novel biomaterials. Macromolecular Bioscience, 6(12), 991-1008.

Prabaharan, M., \& Mano, J. F. (2006b). Liquid crystalline behaviour of chitosan in formic, acetic, monochloroacetic acid solutions. Materials Science Forum, 1010 514-516.

Quijada-Garrido, I., Iglesias-Gonzalez, V., Mazon-Arechederra, J. M., \& BarralesRienda, J. M. (2007). The role played by the interactions of small molecules with chitosan and their transition temperatures. Glass-forming liquids: 1, 2, 3Propantriol (glycerol). Carbohydrate Polymers, 68(1), 173-186.

Rinaudo, M. (2006). Chitin and chitosan: Properties and applications. Progress in Polymer Science, 31(7), 603-632.

Sakurai, K., Maegawa, T., \& Takahashi, T. (2000). Glass transition temperature of chitosan and miscibility of chitosan/poly( $N$-vinyl pyrrolidone) blends. Polymer 41(19), 7051-7056.

Shantha, K. L., \& Harding, D. R. K. (2002). Synthesis and characterisation of chemically modified chitosan microspheres. Carbohydrate Polymers, 48(3), 247-253.

Silva, G. A., Ducheyne, P., \& Reis, R. L. (2007). Materials in particulate form for tissue engineering. 1. Basic concepts. Journal of Tissue Engineering and Regenerative Medicine, 1(1), 4-24

Silva, R. M., Silva, G. A., Coutinho, O. P., Mano, J. F., \& Reis, R. L. (2004). Preparation and characterisation in simulated body conditions of glutaraldehyde crosslinked chitosan membranes. Journal of Materials Science - Materials in Medicine, 15(10), 1105-1112.
Sorlier, P., Denuziere, A., Viton, C., \& Domard, A. (2001). Relation between the degree of acetylation and the electrostatic properties of chitin and chitosan. Biomacromolecules, 2(3), 765-772.

Sorlier, P., Rochas, C., Morfin, I., Viton, C., \& Domard, A. (2003). Light scattering studies of the solution properties of chitosans of varying degrees of acetylation. Biomacromolecules, 4(4), 1034-1040.

Sorlier, P., Viton, C., \& Domard, A. (2002). Relation between solution properties and degree of acetylation of chitosan: Role of aging. Biomacromolecules, 3(6), 1336-1342.

Terbojevich, M., Cosani, A., \& Muzzarelli, R. A. A. (1996). Molecular parameters of chitosans depolymerized with the aid of papain. Carbohydrate Polymers, 29(1), 63-68.

Toffey, A., \& Glasser, W. G. (2001). Chitin derivatives III formation of amidized homologs of chitosan. Cellulose, 8(1), 35-47.

Tuzlakoglu, K., Alves, C. M., Mano, J. F., \& Reis, R. L. (2004). Production and characterization of chitosan fibers and 3-D fiber mesh scaffolds for tissue engineering applications. Macromolecular Bioscience, 4(8), 811-819.

Uragami, T., Matsuda, T., Okuno, H., \& Miyata, T. (1994). Structure of chemicallymodified chitosan membranes and their characteristics of permeation and separation of aqueous-ethanol solutions. Journal of Membrane Science, 88(2-3), 243-251.

Varum, K. M., Anthonsen, M. W., Grasdalen, H., \& Smidsrod, O. (1991). High-field NMR-spectroscopy of partially $N$-deacetylated chitins (chitosans). 1 . Determination of the degree of $\mathrm{N}$-acetylation and the distribution of $\mathrm{N}$-acetyl groups in partially $\mathrm{N}$-deacetylated chitins (chitosans) by high-field NMRspectroscopy. Carbohydrate Research, 211(1), 17-23.

Viciosa, M. T., Dionisio, M., \& Mano, J. F. (2006). Dielectric characterization of neutralized and nonneutralized chitosan upon drying. Biopolymers, 81(3), 149-159.

Viciosa, M. T., Dionisio, M., Silva, R. M., Reis, R. L., \& Mano, J. F. (2004). Molecular motions in chitosan studied by dielectric relaxation spectroscopy. Biomacromolecules, 5(5), 2073-2078.

Yao, K. D., Liu, J., Cheng, G. X., Zhao, R. Z., Wang, W. H., \& Wei, L. (1998). The dynamic swelling behaviour of chitosan-based hydrogels. Polymer International, 45(2), 191-194. 